\title{
Characteristics of hunters and the socio-
}

\section{economic importance of selling game on the} survival of village households in the Kisangani Forest Region (R.D.C.)

\section{Caractéristiques des chasseurs et importance socioéconomique de la vente de gibiers sur la survie des ménages villageois en région forestière de Kisangani (R.D.C)}

Consolate Kaswera Kyamakya ${ }^{1}$, Richard Mendako Kashinda ${ }^{2}$, Kasereka Vitekere ${ }^{3}$

${ }^{1}$ Faculté des Sciences/CSB/UNIKIS

${ }^{2,3}$ Faculté des Sciences UNIKIS

\begin{abstract}
The present study focuses on the description of hunting professionals and intends to determine the contribution of hunting in the survival of village households in the former Province Orientale. It contributes to the in-depth knowledge of the profile of these key players in the commercial bushmeat market and the understanding of the socio-economic context at the first level of the game sales chain. The methods used to achieve the set objectives are summarized first of all in the questionnaire which made it possible to establish the survey form containing the identity of the interviewees and other descriptions, the interview, then the direct observation on the ground. , cost-benefit analysis and chisquare test and Anova.

After tabulation and data processing, it appears that the hunters are all men whose age varies from 20 to 69 years. They come from different ethnic backgrounds and most of them are married and responsible for households. As a level of education, there are among them illiterates, primarians and some graduates.

The results show that bushmeat consumption occurs 1 to 3 times per week in most village households. The average monthly hunter's income estimate is USD 58.18 to meet the multiple daily needs.
\end{abstract}

Keywords-bush meat, profile, hunters, socio-economic importance, hunting income, village hunting, Rubi Tele, Kisangani
Résumé- La présente étude se focalise sur la description des professionnels de la chasse et compte déterminer l'apport de la chasse dans la survie des ménages villageois dans l'ancienne Province Orientale. Elle contribue à la connaissance approfondie du profil de ces acteurs clés du circuit commercial de la viande de brousse et la compréhension du contexte socioéconomique au premier niveau de la filière de vente du gibier. Les méthodes utilisées pour atteindre les objectifs fixés se résument tout d'abord au questionnaire qui a permis d'établir la fiche d'enquête contenant l'identité des enquêtés et d'autres descriptions, l'interview, ensuite l'observation directe sur terrain, l'analyse des coûts-bénéfices et le test de chi-carré et Anova.

Après dépouillement et traitement de données, il ressort que les chasseurs sont tous des hommes dont l'âge varie de 20 à 69 ans. Ils sont issus de différentes ethnies et la plupart d'entre eux sont mariés et responsables de ménages. Comme niveau d'instruction, on compte parmi eux des analphabètes, des primairiens et quelques diplômés.

Les résultats démontrent ensuite que la consommation de la viande de brousse intervient 1 à 3 fois par semaine dans la plupart des ménages villageois. L'estimation de revenu mensuel moyen tiré par un chasseur se fixe à USD 58,18 lui permettant de faire face aux multiples besoins quotidiens. 
Mots clés - viande de brousse, profil, chasseurs, importance socioéconomique, revenus de chasse, chasse villageoise, Rubi-Télé, Kisangani.

\section{INTRODUCTION}

Un commerce florissant de viande de brousse s'accroit ce dernier temps en R.D.C. Plusieurs facteurs sont cités comme causes de ce phénomène. Nous retenons entre autre la demande grandissante en protéines animales (Basa et al., 2017), la crise économique et le sousemploies (Bahuchet, 2000). La chasse se présente comme l'ultime alternative pour les forestiers (Wilkie et al., 2005). L'usage des techniques non écologiques, les armes à feu et le non-respect de la réglementation et textes légaux sur la chasse en vigueur en R.D.C sont des abus régulièrement commis par les villageois en vue de maximiser leurs gaies (Consolate Kaswera et al., 2016, 2017).

De nos jours, quelques données sont disponibles par rapport au flux de viande sur les différents marchés urbains et péri urbains de Kisangani (Belembo et al.,2003 ; Nebesse, 2016). Néanmoins, les publications orientées vers les chasseurs qui du reste sont les acteurs clés du circuit de vente de cette ressource demeurent lacunaires voir inexistantes. Leurs comportements ainsi que les techniques de capture, les modes de conservation et de traitement des carcasses ne restent pas constants puisque le contexte change et ils essayent de s'adapter tant mieux que mal. Les prélèvements se font aussi bien pour l'autosubsistance que pour la vente. La durabilité des activités cynécologiques dépend donc ipso facto du comportement responsable de ces acteurs au niveau primaire du circuit de commercialisation de viande de brousse. Ces professionnels et amateurs de chasse doivent être conscientisés, éduqués, encadrés, écoutés et accompagnés. Cela n'est possible que lorsque leur profil est connu, les motivations les poussant à prélever plus d'espèces ainsi que leurs conditions sociales améliorées.
Nous nous sommes posé trois questions fondamentales à savoir :

$1^{\circ}$ Quel est le profil des chasseurs en région forestière de Kisangani?

$2^{\circ}$ Comment s’y prennent-ils actuellement pour prélever les gibiers?

$3^{\circ}$ Quel est 1'apport de la chasse dans la survie des chasseurs villageois

La présente étudevise tout d'abord à décrire le portrait des chasseurs dans l'ancienne Province Orientale en République Démocratique du Congo, ensuite démontrer la tendance actuelle d'abandonner les pièges traditionnels vers l'usage d'armes à feu plus performants. Enfin, elle établit l'apport socioéconomique de la vente des gibiers sur les ménages au premier niveau de la filière viande de brousse en région forestière de Kisangani.

Comme intérêt, l'étude fournit aux scientifiques et gestionnaires de la biodiversité des données relatives aux chasseurs qui sont des collaborateurs et partenaires en matière de stratégies de gestion durable des ressources faunistiques.

Les hypothèses sont formulées comme suit :

$1^{\circ}$ Les acteurs de chasse proviennent de toutes les couches sociales présentent dans la contrée,

$2^{\circ}$ Les chasseurs font usage de plusieurs techniques pour optimiser les prélèvements.

$3^{\circ}$ L'apport de la chasse s'évalue en termes de consommation alimentaire et des revenus monétaires dans la survie des chasseurs villageois.

\section{Présentation des zones d'études}

Les investigations avaient été menées dans les zones giboyeuses situées sur deux axes routiers à savoir Kisangani-Ituri et Kisangani -Buta. Les villages visités sur la route Ituri en Province de la Tshopo sont Baegofa et Bafwaboli situés respectivement aux points kilométriques 122 et 147 de mai à juillet 2015 (figure1).

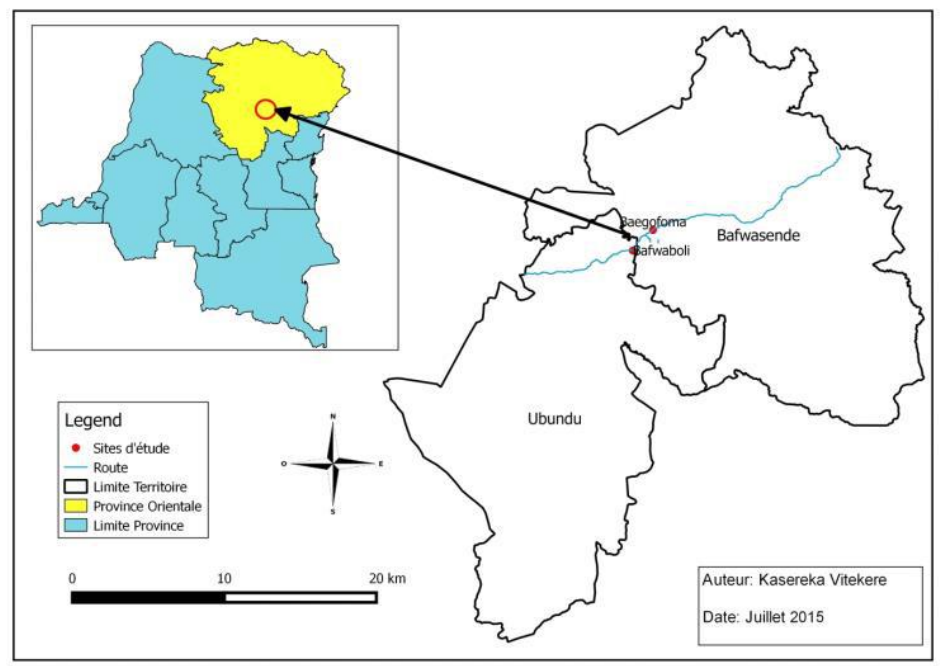

Fig.1: Localisation des sites giboyeux sur la route Ituri 
Sur la route Buta, la prospection avait été menée au sein du Domaine de chasse de Rubi-Télé (DCRT). Pour cette étude, 5 localités ont été retenues. Nous sommes partis d'abord de la localité Baangba (Sukisa/centre), puis deux localités en amont dont Bondeme et Ngbete et deux autres en aval dont Bongbongo et Bobusanga. La sélection des localités a été faite sur base des critères comme l'effectivité de l'activité de chasse dans la localité; la facilité d'accéder à la localité et la distance de la localité par rapport à Baangba/Sukisa, étant donné que cette localité a constitué notre point de départ.

Le DCRT touche essentiellement cinq entités territoriales administratives dont les Territoires d'Aketi, Bambesa et
Buta situés dans la Province du Bas-Uélé d'une part, et les Territoires de Banalia et Basoko qui se localisent dans la Province de la Tshopo d'autre part. Ce domaine de chasse s'étale donc sur 8 secteurs ou collectivités dont certains se trouvent dans la Province du Bas-Uélé : Bayeu-Bogbama, Mabinza, Makere II, Mongazulu et Yoko, et d'autres dans la Province de la Tshopo : Baboa de Kole, Wahanga, et Yamandundu. Ceci justifie la diversité ethnique constatée lors de l'échantillonnage.

Les cinq localités du DCRT visitées sont Baangba, Bongbongo, Bobusanga, Bondeme et Ngbete (figure 2). La collecte des données dans ce site était effectuée au cours du mois de juin 2016.

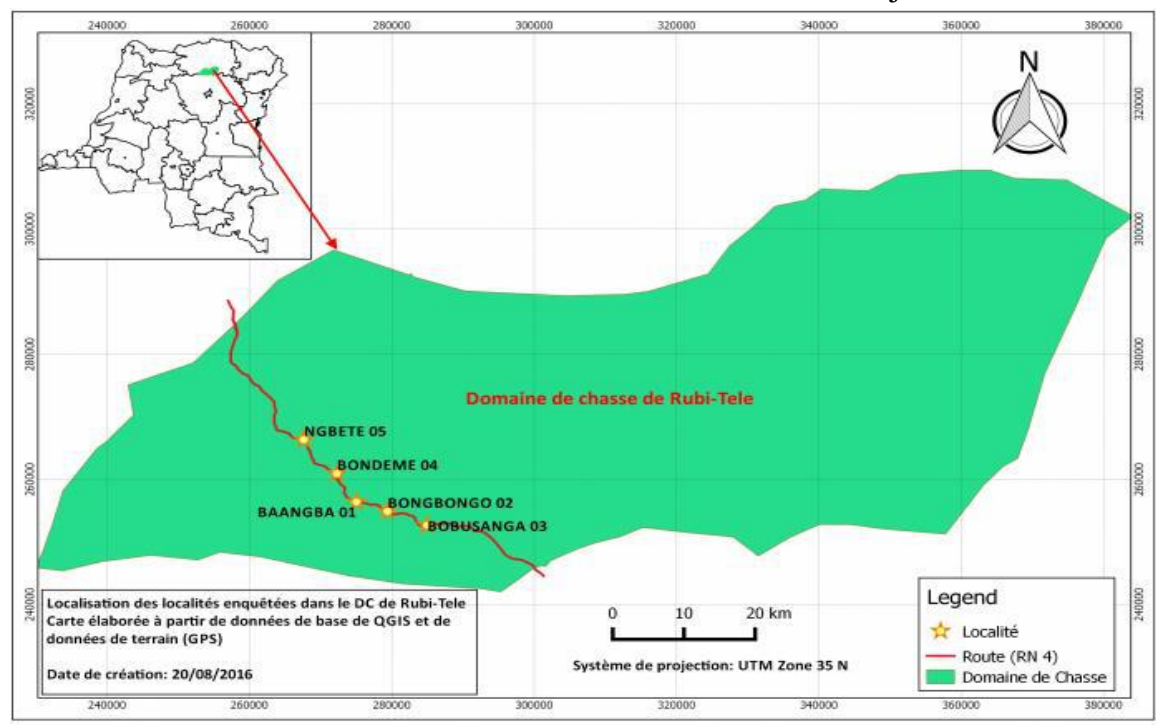

Fig.2: Domaine de chasse de Rubi-Télé (DCRT)

\section{METHODOLOGIES}

Nous avions capitalisé les informations données par les revendeuses de viande de brousse au marché central de Kisangani lors d'un pré enquête diligenté avant la récolte des données proprement dite.

Un guide d'interview a été mis à place et soumis aux chasseurs présents dans les villages. Les questions étaient interprétées en Kiswahili ou Lingala lors de l'administration. L'interview était complétée par des observations de terrain, un suivi des chasseurs pendant plus ou moins deux mois et des entretiens auprès de la population locale.

Compte tenu de la difficulté de disposer des listes de tous les chasseurs dans les localités retenues, nous avons procédé par la technique de boule de neige (utilisation de personnes comme source d'identification d'unités additionnelles) pour le choix de l'échantillon. Dans ce type d'échantillon, appelé aussi échantillon par réseau, les individus sont sélectionnés en fonction de leurs liens avec un «noyau» d'individus. On se base par exemple sur les réseaux sociaux, les amitiés, les relations d'affaires pour recruter de nouveaux sujets (Fortin, 2008).

\section{Traitement des données}

Les données récoltées à partir de l'enquête ont été parcourues, dépouillées, codifiées et saisies sur Microsoft Excel 2010, à partir duquel une base des données a été constituée. Partant de cette dernière, quelques calculs ont été appliqués en vue de déterminer certaines statistiques (fréquences absolues et relatives, moyennes, écart-type, valeur maximale, valeur minimale, etc). Le test statistique Anova a été appliqué pour la comparaison des moyennes. L'interprétation des résultats obtenue (p-value) au seuil de signification $(\alpha=0,05=5 \%)$. Si $p$-value $>\alpha$ : pas de différence significative entre les moyennes; alors que si p-value $<\alpha$ : la différence est significative entre les moyennes.

Le test de l'indépendance du chi-carré pour analyser la relation entre 2 variables qualitatives. Si p-value $>$ au seuil de 5\%: pas de différence significative, les deux variables sont indépendantes; tandis que si p-value < $\alpha$ : il existe une différence significative, les deux variables ne sont pas indépendantes.

Détermination de l'apport de la chasse dans la survie des chasseurs 
Pour la fréquence de consommation, nous avons évalué, au cours d'une semaine le nombre de fois que le ménage consomme la viande de brousse.

Pour estimer les revenus monétaires issus de la vente des produits de chasse, nous sommes partis d'abord de l'estimation des coûts mensuels de la chasse, puis de l'estimation des recettes mensuelles issues de la vente des gibiers. Afin l'estimation de Revenu monétaire (Profit) déterminée par la différence entre les recettes et les coûts mensuels de chasse.

Les coûts retenus et estimés après entretiens avec les chasseurs sont notamment ceux liés à l'achat des munitions (cartouches), la ration mensuelle ainsi que l'achat d'autres équipements (piles de torche) par mois.

Coût Total $(\mathrm{CT})=$ Achat munitions + Ration + Achat autres équipements

Les recettes étaient obtenues en multipliant le prix moyen unitaire par le nombre des gibiers estimés capturés par mois.

Recettes/Espèce $=$ Nbre des gibiers $x$ PMU

Recettes Totales $(\mathrm{RT})=\sum$ Recettes/Espèces

Revenu monétaire mensuel issu de la vente des produits de chasse :

Revenu mensuel Total (Profit) $=$ RT $-\mathrm{CT}$

Revenu mensuel moyen=Revenu mensuel Total/n

$\mathrm{n}=$ taille d'échantillon ( 52 chasseurs enquêtés)

Rentabilité économique de la chasse (RE) : le résultat (Revenu/Profit) comparé au Chiffre d'affaires (Recettes Totales)

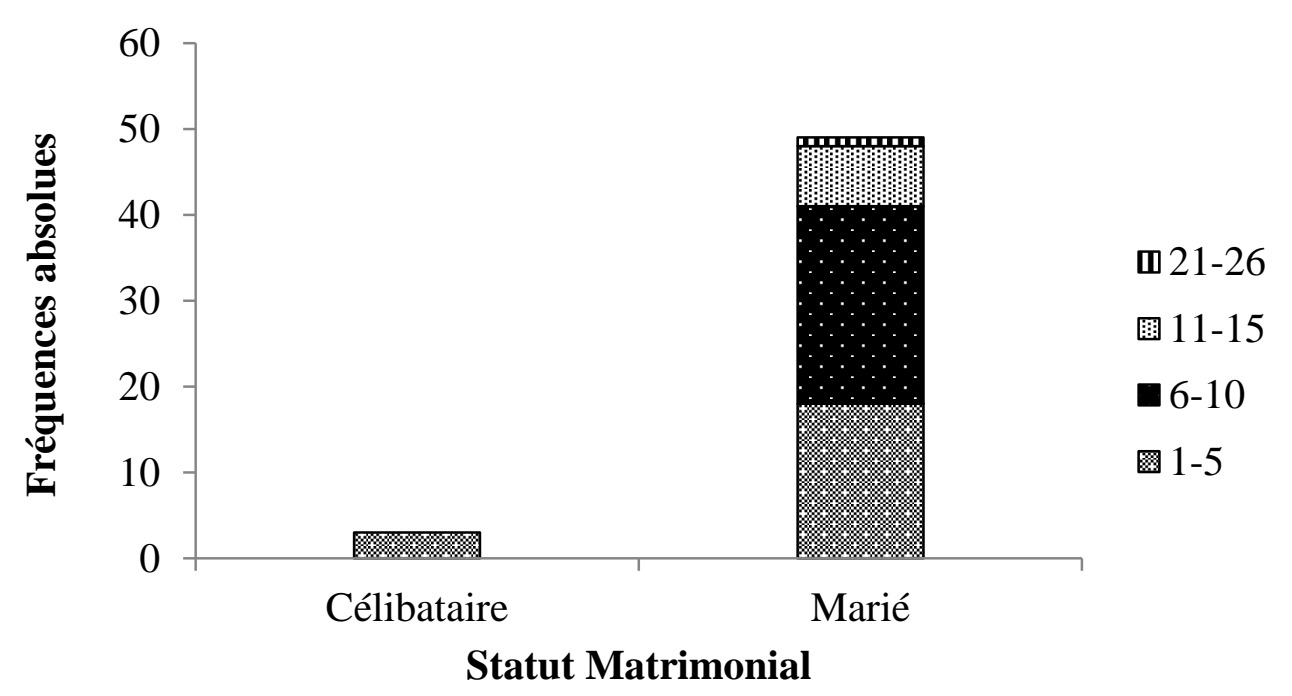

Fig.3: Répartition des chasseurs en fonction de statut matrimonial et taille de ménage

$$
\begin{aligned}
\mathbf{R E}= & \frac{\text { Revenu mensuel Total }(\text { Profit }) * 100}{\text { Chiffre d'affaires }(\text { Recettes Totales })} \\
& \text { ou } \frac{\text { Revenu mensuel moyen } * 100}{\text { Recettes mensuelles moyennes }}
\end{aligned}
$$

\section{RESULTS}

\section{Profil sociodémographique des chasseurs}

$\mathrm{Au}$ total 94 chasseurs exclusivement hommes ont été questionnés dans les deux zones giboyeuses retenues. Sur la route Ituri, 21 chasseurs étaient inventoriés au village Baegofoma et 21 autres à Bafwaboli. Sur la route Buta, 52 chasseurs ont été questionnés en raison de 10 chasseurs par chaque localité: Baangba, Bondeme, Ngbete et Bongbongo, alors que 12 autres ont été enquêtés dans la localité Bobusanga.

1.1. Statut matrimonial des chasseurs et taille des ménages

Parmi les chasseurs interviewés 5,7\% sont célibataires et 94,3\% mariés. Les célibataires vivent dans les ménages composés de 1 à 5 individus. Par contre pour la catégorie des chasseurs mariés $36,7 \%$ ont une taille de ménage composé de 1 à 5 individus, 46,9\% chasseurs ont une taille de ménage constitué de 6 à 10 individus, $14,4 \%$ en ont constitué de 11 à 15 individus et enfin $2 \%$ gèrent une taille de ménage allant jusque plus de 20 individus (figure 3). 
Partant de l'âge, du statut matrimonial et de la taille des ménages, on comprend que la majorité de chasseurs exerce cette activité pour l'autosubsistance des ménages dans le cadre de la responsabilité familiale.

\subsection{Activités principales des chasseurs enquêtés}

L'activité principale pour la majorité des chasseurs enquêtés est l'agriculture, tandis que les restes pratiquent la chasse comme activité principale (figure 4).

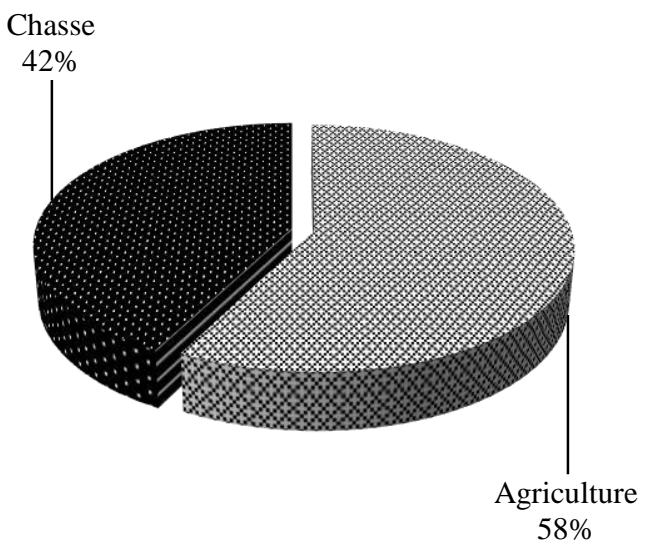

Fig.4: Répartition des chasseurs par activité principale

L'agriculture semble être l'activité principale des populations vivant en périphérie et dans les zones étudiées. Elle est cependant peu développée suite aux superficies limitées et une faible diversité des spéculations. Elle n'est pas susceptible de subvenir aux besoins de cette population en termes de revenus car les filières agricoles ne sont pas encore suffisamment développées (Pro-Routes, 2013). La collecte saisonnière des produits forestiers non ligneux tels que du miel, des chenilles, des escargots et la pêche est également pratiquée.

\subsection{Age des chasseurs}

L'âge des chasseurs s'étend de 20 à 69 ans d'après le tableau (1). La majorité d'enquêtés se retrouve dans la tranche d'âge comprise entre 20-49 ans caractérisée par une grande responsabilité familiale.

Tableau.1 : Effectifs par intervalle d'âge des chasseurs

\begin{tabular}{|c|c|c|c|c|c|c|}
\hline $\begin{array}{l}\text { Intervalle } \\
\text { d'âge }\end{array}$ & $\begin{array}{l}\text { Effectifs } \\
\text { chasseurs } \\
\text { Route Ituri }\end{array}$ & $\%$ & $\begin{array}{l}\text { Effectifs } \\
\text { chasseurs } \\
\text { DCRT }\end{array}$ & $\%$ & $\begin{array}{l}\text { Total } \\
\text { chasseurs }\end{array}$ & $\%$ \\
\hline $20-29$ & 9 & 21,4 & 14 & 26,9 & 23 & 24,4 \\
\hline 30-39 & 13 & 30,9 & 19 & 36,5 & 32 & 34 \\
\hline 40-49 & 11 & 26,1 & 13 & 25 & 24 & 25,5 \\
\hline $50-59$ & 8 & 19 & 5 & 9,6 & 13 & 13,8 \\
\hline 60-69 & 1 & 2,3 & 1 & 1,9 & 2 & 2,1 \\
\hline
\end{tabular}

Il découle de ce tableau que l'âge des chasseurs varie de 20 à 69 ans. La majorité des chasseurs actifs se retrouve entre 20 et 49 ans, avec une prédominance de la tranche d'âge 30-39 ans qui a fourni $34 \%$ de l'effectif inventorié sur les deux sites.

\subsection{Niveau d'instruction des chasseurs par intervalle d'âge}

Il ressort des enquêtes qu'au sein de l'intervalle d'âge 20 - 49 ans, lequel intervalle regroupe la majorité des chasseurs actifs, $41,1 \%$ ont un niveau secondaire d'instruction, $34,6 \%$ sont d'un niveau primaire et 7,6\% analphabètes. Par ailleurs, pour l'intervalle d'âges 50-69 ans, 9,6\% sont d'un niveau d'études primaires et 1,9\% d'un niveau d'études secondaires.

Le test chi-carré appliqué pour vérifier la différence de la répartition des chasseurs par classe d'âges et par niveau d'instruction a montré une différence non significative, les deux variables sont indépendantes $\left(X^{2}=4.26\right.$, degré de liberté $(\mathrm{ddl})=2$, $\mathrm{p}$-value $=0,12>\alpha=0,05$, différence non significative). 


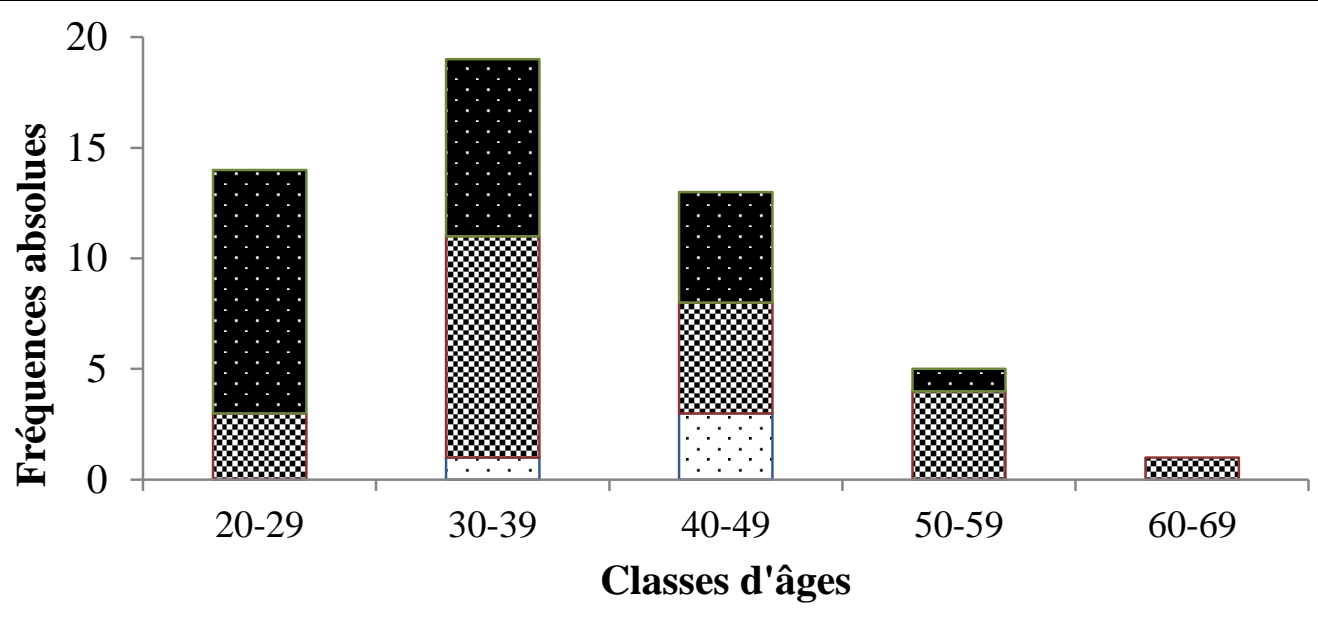

$\square$ Analphabète $\mathrm{B}$ Primaire Secondaire

Fig.5: Niveau d'études des enquêtés par intervalle d'âge

1.5. Ages des chasseurs et techniques de chasse utilisées

Pour l'intervalle d'âges 20- 49 ans, 46,1\% recourent à l'association pièges et fusil de fabrication locale (PFFL) alors que $42,3 \%$ utilisent exclusivement les fusils de fabrication locale (FFL) pour un bon rendement de l'activité. En outre, pour la catégorie des chasseurs avancés en âge (50-69 ans), 5,7\% recourent aux FFL et 6 $\%$ recourent à la technique mixte PFFL. Le constat est que de plus en plus les chasseurs considèrent que les pièges seuls ne sont pas rentables et ne les font pas habituellement. C'est pour cette raison que dans le cadre de cette recherche, nous avons eu seulement à analyser les aspects socioéconomiques de la chasse au fusil. Le test d'indépendance du chi-carré appliqué démontre que la répartition des chasseurs par classe d'âges et techniques utilisées ne diffère pas significativement, c'est-à-dire que les deux variables sont indépendantes $\left(\mathrm{X}^{2}=0\right.$, ddl $=1, \mathrm{p}$ value $=1>\alpha=0,05$; différence non significative).

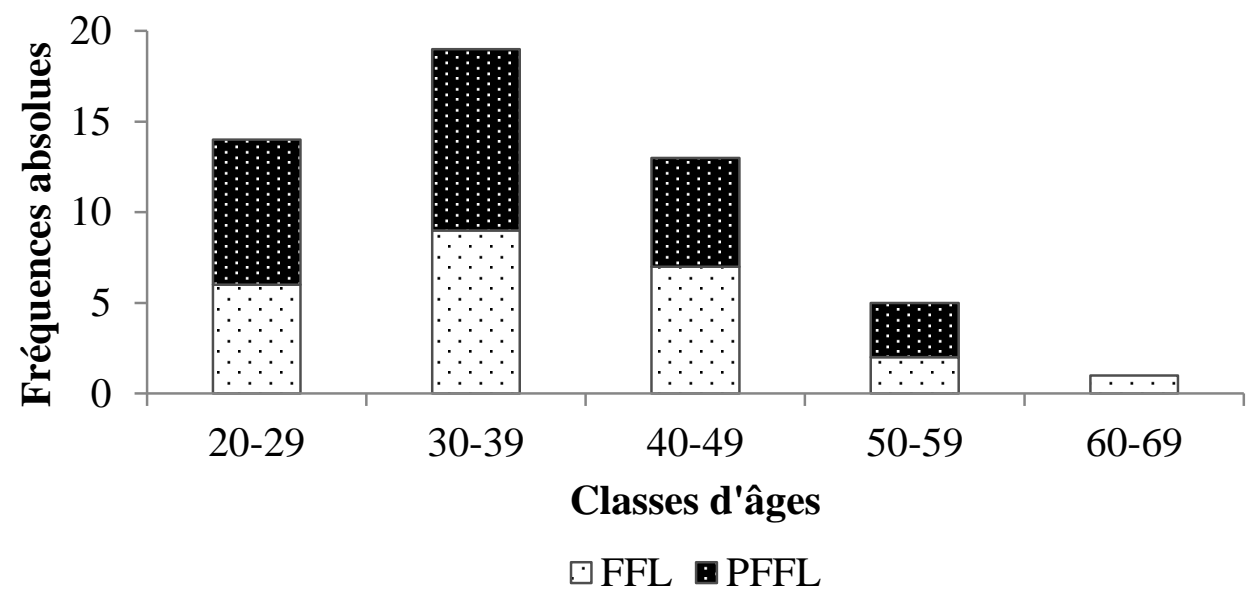

Fig.6: Répartition des chasseurs par classe d'âges et techniques utilisées

1.6. Ages des chasseurs et années d'expérience
d'activité
Il ressort de la figure (figure 7 ) ci-dessous que les
chasseurs actifs ( 20 à 49 ans) ont une expérience de 1 à
30 ans d'une part, et ceux avancés en âge ( 50 à 69 ans) en
ont de 31 à 50 ans d'autre part. La répartition des chasseurs en fonction de l'âge et expérience diffère significativement, les deux variables sont dépendantes. L'expérience dépend de l'âge du chasseur $\left(\mathrm{X}^{2}=42.66\right.$, ddl $=1$, p-value $=6.499 \mathrm{e}-11<\alpha=0,05$, différence significative). 


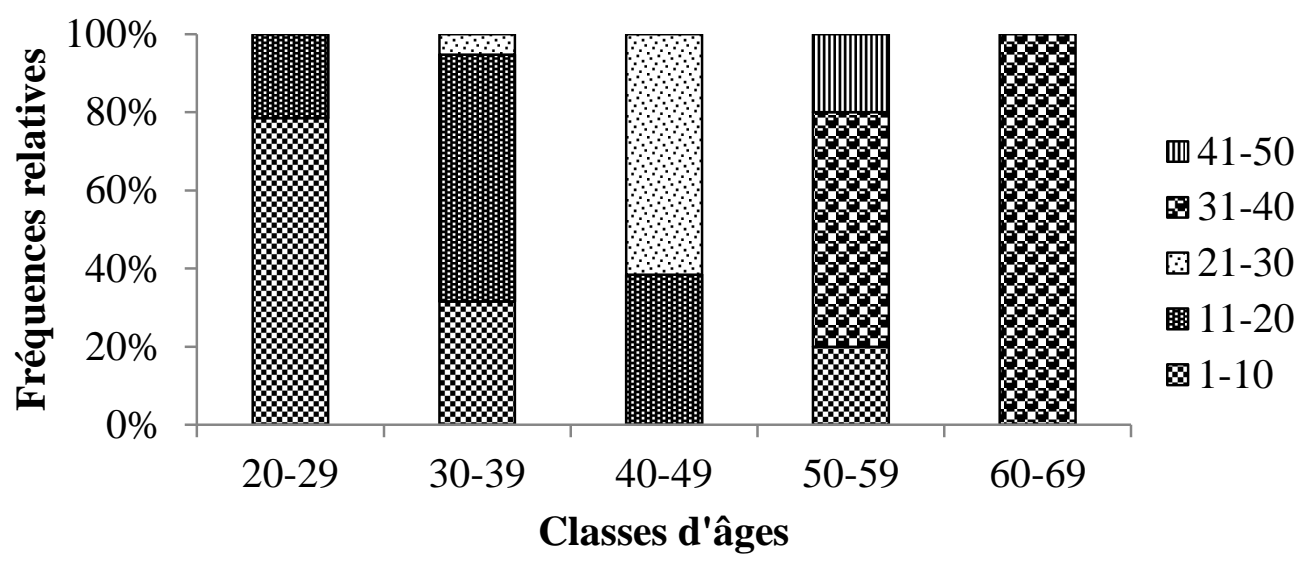

Fig.7: Répartition des chasseurs en fonction de l'âge et expérience

\subsection{Appartenance ethnique}

Les chasseurs recensés au sein de nos sites d'études proviennent de groupes ethniques variés. La majorité des chasseurs recensés n'est pas faite que d'autochtones, d'autres sont originaires des provinces voisines. A Baegofoma, les ethnies prédominantes sont: les Topoke $(38,09 \%)$, les Mongo (19,04\%) et les Bali (9,52\%) tandis que les ethnies minoritaires sont constituées de Kumu, et Ngelema (1\%). A Bafwaboli les chasseurs sont majoritairement Mbole (19,04\%). Pour le DCRT, les
Bowa représentent 51,9\%, suivis des Ngelema 44,2\%, des Zande $1,9 \%$ et des Ngbeto 1,9\%.

2. Apport de la chasse dans la survie des chasseurs villageois du Domaine de chasse de Rubi - Télé

2.1. Espèces chassées, prix moyens unitaires (PMU) et leurs statuts

Les informations relatives aux taxa régulièrement chassés dans ce domaine, le prix moyen unitaire par espèce et le statut de protection font l'objet du tableau ci dessous.

Tableau.2 : Fréquences des espèces chassées, PMU et leurs statuts

\begin{tabular}{|c|c|c|c|c|c|c|}
\hline \multirow[t]{2}{*}{$\mathbf{N}^{\circ}$} & \multicolumn{2}{|l|}{ Espèces } & \multirow{2}{*}{$\begin{array}{l}\text { Fréq. } \\
\text { relatives }\end{array}$} & \multirow[t]{2}{*}{ PMU(\$) } & \multicolumn{2}{|c|}{ Statuts de protection } \\
\hline & Noms scientifiques & $\begin{array}{l}\text { Noms } \\
\text { vernaculaires }\end{array}$ & & & RDC & UICN \\
\hline 1 & $\begin{array}{l}\text { Cephalophus monticola } \\
\text { Thunberg, } 1789\end{array}$ & Mboloko & $98 \%$ & 3,62 & NP & LC \\
\hline 2 & $\begin{array}{l}\text { Cephalophus dorsalis } \\
\text { Gray, } 1846\end{array}$ & Koto & $83 \%$ & 18,96 & NP & LC \\
\hline 3 & $\begin{array}{l}\text { Cercopithescus ascanius } \\
\text { Audebert, } 1799\end{array}$ & Makako & $79 \%$ & 4,06 & NP & VU \\
\hline 4 & $\begin{array}{l}\text { Atherurus africanus } \\
\text { Gray, } 1842\end{array}$ & Ndjiko & $50 \%$ & 2,91 & NP & LC \\
\hline 5 & $\begin{array}{l}\text { Potamocherus porcus } \\
\text { Linnaeus, } 1758\end{array}$ & Ngulu & $35 \%$ & 34,04 & PP & LC \\
\hline 6 & $\begin{array}{l}\text { Cephalophus nigrifons } \\
\text { Gray, } 1871\end{array}$ & Mbengele & $33 \%$ & 15,01 & NP & LC \\
\hline 7 & $\begin{array}{l}\text { Cricetomys emini } \\
\text { Waterhouse, } 1840\end{array}$ & Motomba & $31 \%$ & 1,40 & NP & LC \\
\hline 8 & Cephalophus sylvicultor & Mulimbu & $8 \%$ & 31,58 & & LC \\
\hline 9 & $\begin{array}{l}\text { Hyemoschus aquaticus } \\
\text { Ogilby, } 1841\end{array}$ & Elebé & $8 \%$ & 10,26 & PP & LC \\
\hline 10 & $\begin{array}{l}\text { Tragelaphus spekei } \\
\text { Spek, } 1863\end{array}$ & Mbulimasuwa & $4 \%$ & 52,63 & PP & LC \\
\hline 11 & $\begin{array}{l}\text { Colobus guereza } \\
\text { Sclater, } 1860\end{array}$ & Magistrat & $4 \%$ & 6,84 & TP & $\mathrm{EN}$ \\
\hline 12 & Pan troglodytes & Sokomoto & $4 \%$ & 42,11 & TP & EN \\
\hline
\end{tabular}


Légende :

\section{Statuts selon l'UICN}

EN : Endangered (en danger)

LC : Least Concern (Préoccupation mineure)

VU : Vulnérable (Vulnérable)

2.2. Consommation de la viande de brousse par les ménages des chasseurs villageois

Le tableau ci-dessous stipule que plus de la moitié des enquêtés dans les localités visitées affirment avoir
TP : Totalement Protégée

PP : Partiellement Protégée

NP : Non Protégée

Statuts selon la loi Congolaise (RDC)

consomme la viande de brousse une a trois fois par semaine alors que 5 la consomme rarement. Le chasseur prélève la faune sauvage pour deux motifs essentiels, l'autosubsistance et le lucre.

Tableau.3 : Fréquence de consommation de la viande de gibier par semaine

\begin{tabular}{|c|c|c|c|c|c|c|}
\hline $\begin{array}{ll}\text { Fréq. } & \text { de } \\
\text { cons/sem. } & \end{array}$ & Baangba & Bongbongo & Bobusanga & Bondeme & Ngbete & Total \\
\hline 1 fois & 4 & 1 & 4 & 1 & 6 & 16 \\
\hline 2 fois & 1 & 5 & 5 & 6 & 3 & 20 \\
\hline 3 fois & 1 & 3 & 0 & 0 & 1 & 5 \\
\hline 4 fois & 1 & 0 & 0 & 1 & 0 & 2 \\
\hline 5 fois & 1 & 0 & 0 & 0 & 0 & 1 \\
\hline 6 fois & 1 & 1 & 0 & 0 & 0 & 2 \\
\hline Chaque jour & & 0 & 1 & 0 & 0 & 1 \\
\hline Rarement & 1 & 0 & 2 & 2 & 0 & 5 \\
\hline Total & 10 & 10 & 12 & 10 & 10 & 52 \\
\hline
\end{tabular}

\subsection{Estimation des coûts mensuels de chasse}

Les principaux coûts de chasse identifiés lors de nos enquêtes dans les 5 localités du DCRT sont notamment ceux liés à l'achat des cartouches, la ration et d'autres équipements (piles et torches). Le prix moyen d'une cartouche est estimé à $\$ 1,58$. Ainsi pour un cumul de 99 expéditions par mois, l'estimation faite sur base des entretiens avec les 52 chasseurs de 5 localités enquêtées, il se dégage des charges relatives à l'achat de cartouches de $\$ 915,79$ pour la ration $\$ 159,84$ alors que celles relatives à l'achat d'autres équipements sont estimées à \$ 90,00 .

Tableau.4 : Estimation des coûts mensuels de chasse par localités

\begin{tabular}{|c|c|c|c|c|c|c|c|c|}
\hline Localités & Nbre Expédition & Cartouches & \multicolumn{2}{|c|}{ Ration } & \multicolumn{2}{|c|}{ Autres équipements } & \multicolumn{2}{|c|}{ Total } \\
\hline Baangba & 18 & $\$ \quad 153,16$ & $\$$ & 24,95 & $\$$ & 15,79 & $\$$ & 193,89 \\
\hline Bongbongo & 20 & 192,63 & $\$$ & 43,79 & $\$$ & 23,16 & $\$$ & 259,58 \\
\hline Bobusanga & 25 & $\$ \quad 257,37$ & $\$$ & 42,74 & $\$$ & 21,05 & $\$$ & 321,16 \\
\hline Bondeme & 20 & 183,16 & $\$$ & 27,32 & $\$$ & 15,26 & $\$$ & 225,74 \\
\hline Ngbete & 16 & 129,47 & $\$$ & 21,05 & $\$$ & 14,74 & $\$$ & 165,26 \\
\hline Total & 99 & $\$ \quad 915,79$ & $\$$ & 159,84 & $\$$ & 90,00 & $\$$ & 1165,63 \\
\hline
\end{tabular}

\subsection{Estimation des Recettes brutes issues de la vente} des produits de chasse

Le présent tableau met en lumière l'estimation des recettes brutes mensuelles issues de la vente de viande de brousse. Il en ressort donc le prix moyen unitaire par carcasse boucanée, le nombre des gibiers prélevés par espèce débouchant à une estimation des recettes brutes par espèces puis un total général des recettes brutes par localités. En effet, dans la localité Baangba, il se dégage une estimation de 70 gibiers des espèces confondues prélevés valant une somme de \$673.02, à Bongbongo 92 gibiers pour une valeur monétaire de $\$ 1,022.14$, à Bobusanga 124 gibiers ont été estimés comme prélèvement mensuel générant des recettes brutes de $\$$ $1,155.80 ; 93$ gibiers à Bondeme valant des recettes brutes estimées à \$ 794.49 et enfin, dans la localité de Ngbete, l'estimation des prélèvements mensuels des gibiers des espèces confondues se fixent à 63 gibiers générant des recettes brutes de $\$ 545.58$. 
Tableau.5: Recettes brutes de vente par localités

\begin{tabular}{|c|c|c|c|c|c|c|c|c|c|c|c|c|}
\hline \multirow{2}{*}{$\begin{array}{l}\mathbf{N} \\
\circ\end{array}$} & \multirow[b]{2}{*}{ Espèces } & \multirow[b]{2}{*}{$\begin{array}{l}\text { PUM/carcas } \\
\text { se }\end{array}$} & \multicolumn{2}{|c|}{ Baangba } & \multicolumn{2}{|c|}{ Bongbongo } & \multicolumn{2}{|c|}{ Bobusanga } & \multicolumn{2}{|c|}{ Bondeme } & \multicolumn{2}{|c|}{ Ngbete } \\
\hline & & & $\begin{array}{l}\text { Nbr } \\
\text { e }\end{array}$ & Recettes & $\begin{array}{l}\text { Nbr } \\
\text { e }\end{array}$ & $\begin{array}{l}\text { Recette } \\
\text { S }\end{array}$ & $\begin{array}{l}\text { Nbr } \\
\text { e }\end{array}$ & Recettes & $\begin{array}{l}\text { Nbr } \\
\text { e }\end{array}$ & $\begin{array}{l}\text { Recette } \\
\text { S }\end{array}$ & $\begin{array}{l}\text { Nbr } \\
\text { e }\end{array}$ & $\begin{array}{l}\text { Recette } \\
\text { S }\end{array}$ \\
\hline 1 & $\begin{array}{l}\text { Cercopithecus } \\
\text { ascanius }\end{array}$ & $\$ 4,06$ & 21 & $\$ 85,36$ & 34 & $\begin{array}{l}\$ \\
138,21\end{array}$ & 45 & $\$ 182,92$ & 46 & $\begin{array}{l} \\
186,99\end{array}$ & 24 & $\begin{array}{l}\$ \\
97,56\end{array}$ \\
\hline 2 & $\begin{array}{l}\text { Cephalophus } \\
\text { monticola }\end{array}$ & $\$ 3,62$ & 22 & $\$ 79,72$ & 26 & $\$ 94,21$ & 28 & $\$ 101,46$ & 20 & $\begin{array}{l}\$ \\
72,47\end{array}$ & 19 & $\begin{array}{l}\$ \\
68,85\end{array}$ \\
\hline 3 & $\begin{array}{l}\text { Cephalophus } \\
\text { dorsalis }\end{array}$ & $\$ 18,96$ & 26 & $\$ 492,92$ & 22 & $\begin{array}{l}\$ \\
417,09\end{array}$ & 36 & $\begin{array}{l} \\
682,51\end{array}$ & 26 & $\begin{array}{l}\$ \\
492,92\end{array}$ & 20 & $\begin{array}{l}\$ \\
379,17\end{array}$ \\
\hline 4 & $\begin{array}{l}\text { Cephalophus } \\
\text { nigrifons }\end{array}$ & $\$ 15,01$ & 1 & $\$ 15,01$ & 0 & $\$$ & 12 & $\begin{array}{l}\$ \\
180,17\end{array}$ & 0 & $\$$ & 0 & $\$$ \\
\hline 5 & $\begin{array}{l}\text { Cephalophus } \\
\text { sylvicultor }\end{array}$ & $\$ 31,58$ & 0 & $\$$ & 2 & $\begin{array}{l}\$ \\
63,16\end{array}$ & 0 & $\$$ & 0 & $\$$ & 0 & $\$$ \\
\hline 6 & $\begin{array}{l}\text { Tragelaphus } \\
\text { spekey }\end{array}$ & $\$ 52,63$ & 0 & $\begin{array}{l} \\
-\end{array}$ & 2 & $\begin{array}{l}\$ \\
105,26\end{array}$ & 0 & $\begin{array}{l}\$ \\
-\end{array}$ & 0 & $\$$ & 0 & $\$$ \\
\hline 7 & $\begin{array}{l}\text { Atherurus } \\
\text { africanus }\end{array}$ & $\$ 2,91$ & 0 & $\begin{array}{l} \\
-\end{array}$ & 0 & $\begin{array}{l}\$ \\
- \\
\end{array}$ & 3 & $\begin{array}{l} \\
8,74 \\
\end{array}$ & 0 & $\$$ & 0 & $\$$ \\
\hline 8 & $\begin{array}{l}\text { Potamochoerus } \\
\text { porcus }\end{array}$ & $\$ 34,04$ & 0 & $\begin{array}{l}\$ \\
-\end{array}$ & 6 & $\begin{array}{l}\$ \\
204,21\end{array}$ & 0 & $\begin{array}{l} \\
\end{array}$ & 0 & $\$$ & 0 & $\$$ \\
\hline 9 & Pan troglodytes & $\$ 42,11$ & 0 & $\begin{array}{l}\$ \\
-\end{array}$ & 0 & $\begin{array}{l}\$ \\
- \\
\end{array}$ & 0 & $\begin{array}{l}\$ \\
-\end{array}$ & 1 & $\$ 42,11$ & 0 & $\begin{array}{l} \\
-\end{array}$ \\
\hline & TAL & & 70 & $\$ 673,02$ & 92 & $\begin{array}{l}\$ \\
1022,14\end{array}$ & 124 & $\$ 155,80$ & 93 & $\begin{array}{l}\$ \\
794,49\end{array}$ & 63 & $\begin{array}{l}\$ \\
545,58\end{array}$ \\
\hline
\end{tabular}

Dans la localité Baangba, il se dégage une estimation de 70 gibiers toutes espèces confondues prélevés valant une somme de USD 673,02; à Bongbongo 92 gibiers pour une valeur monétaire de USD 1 022,14; à Bobusanga 124 gibiers ont été estimés comme prélèvement mensuel générant des recettes de USD 1155,80 ; 93 gibiers à Bondeme valant des recettes estimées à USD 794,49 et enfin, dans la localité de Ngbete, un prélèvement estimé à 63 gibiers générant des recettes de USD 545,58.

La différence n'est pas significative entre les nombres moyens des gibiers prélevés dans les localités (Annexe 5) $(\mathrm{p}$-value $=0,17>0,05)$.

\subsection{Estimation des Coûts Totaux et Recettes Totales} de la chasse

Les coûts totaux mensuels ont été estimés à USD 1165,63 tandis que les recettes totales mensuelles à USD 4 191,01 (pour les 52 chasseurs). Pour un nombre moyen d'expédition de 1,90 ; le coût moyen mensuel est estimé à USD 22,42 et des recettes mensuelles moyennes sont estimées à USD 80,60. Il sied de souligner que la différence entre les coûts moyens des localités n'est pas significative ( $p=0,16>\alpha=0,05)$. C'est le cas aussi pour les recettes moyennes des localités, $(p=0,37>\alpha=0,05)$.

Tableau.6: Coûts Totaux et Recettes Totales de la chasse (en USD)

\begin{tabular}{lc}
\hline Coûts de chasse & \\
\hline Baangba & 193,89 \\
\hline Bongbongo & 259,58 \\
Bobusanga & 321,16 \\
Bondeme & 225,74 \\
Ngbete & 165,26 \\
Coûts totaux mensuels de chasse & $\mathbf{1 1 6 5 , 6 3}$ \\
Recettes brutes & 673,02 \\
Baangba & 1022,14 \\
Bongbongo & 1155,80 \\
\hline Bobusanga & 794,49 \\
\hline Bondeme & \\
\hline
\end{tabular}




\section{Ngbete}

Recettes totales mensuelles
545,58

4191,01

\subsection{Estimation de Revenu/Profit mensuel moyen}

Le revenu total de l'activité de chasse pour les 52 chasseurs est estimé à USD $3 \mathbf{0 2 5 , 3 8}$, un chasseur tire en moyenne un revenu mensuel estimé à USD 58,18.

Partant des résultats de cette étude ponctuelle, la chasse villageoise dans le DCRT apparaît comme une activité financièrement rentable, ainsi la rentabilité économique est estimée à 72,19\% (tableau 7). Ceci dépend de la diversification des stratégies de chasse. Il est souhaitable que des études des longues durées soient conduites, en vue de saisir les fluctuations de la rentabilité de cette activité dans le long terme.

Tableau.7: Revenu mensuel moyen issu de la vente des produits de la chasse (en USD)

\begin{tabular}{|l|l|}
\cline { 2 - 2 } \multicolumn{1}{c|}{} & Valeur monétaire \\
\hline Coûts totaux mensuels de chasse (A) & 1165,63 \\
\hline Recettes Totales mensuelles(B) & 4191,01 \\
\hline Revenu/Profit mensuel Total $(\mathrm{C})=(\mathrm{B})-(\mathrm{A})$ & 3025,38 \\
\hline Revenu/Profit moyen mensuel $(\mathrm{D})=(\mathrm{C}) / 52$ & 58,18 \\
\hline Rentabilité économique $(\mathrm{E})=(\mathrm{C}) * 100 /(\mathrm{B})$ & $72,19 \%$ \\
\hline
\end{tabular}

\subsection{Affectation des revenus de l'activité de chasse}

Partant du tableau suivant, les affectations sont classées selon l'ordre décroissant des fréquences par catégorie d'affectations. Ainsi l'achat de condiments vient en première position avec une fréquence relative de citations de $71 \%$, ensuite vient la scolarisation des enfants avec
$67 \%$, puis les frais liés aux soins de santé avec 58\%, l'habillement avec 50\%. En outre l'achat des produits cosmétiques a une fréquence relative de citations de 19\%, et enfin les chasseurs affectant une partie de leur revenu à l'alcool représentent une fréquence relative de $4 \%$.

Tableau.8: Fréquences des affections de revenu de chasse

\begin{tabular}{|l|l|l|}
\hline Affectations & Fréquences absolues & Fréquences absolues \\
\hline Achat Condiments & 37 & $71 \%$ \\
\hline Scolarisation des enfants & 35 & $67 \%$ \\
\hline Soins de santé & 30 & $58 \%$ \\
\hline Habillement & 26 & $50 \%$ \\
\hline Produits cosmétiques & 10 & $19 \%$ \\
\hline Alcool & 2 & $4 \%$ \\
\hline
\end{tabular}

\section{DISCUSSIONS}

1. Du profil sociodémographique des chasseurs et moyens d'usage

De par les enquêtes menées dans les sites retenus, l'activité de chasse est masculine et pratiquée par des chasseurs actifs (20 à 49 ans), dont $52 \%$ ont un niveau secondaire d'instruction. Il a toujours été avantageux de sensibiliser et collaborer avec des partenaires qui savent lire et écrire. Les gestionnaires des ressources naturelles doivent capitaliser cet atout lors de l'élaboration de divers programmes de sensibilisation et éducation environnementale. Ces chasseurs recourent aux fusils de fabrication locale et des pièges, avec un accent prononcé de recours aux fusils de fabrication locale. L'association calibre 12 avec le chien tel que signalé par les chasseurs semble plus intéressante vu que les animaux sont d'abord dénichés par le chien puis en tentant de se sauver l'arme intervient. En fait, l'arme à calibre 12 est le fusil fabriqué localement le plus utilisé d'après les enquêtés et nos observations lors d'un suivi des chasseurs dans les zones www.ijeab.com d'études.

L'expérience d'activité varie entre 1 à 30 ans. La plupart de ces chasseurs sont mariés et combinent la chasse à l'agriculture.

Endezoumou (2012) a constaté que la chasse était une activité essentiellement masculine dans l'Unité forestière d'Aménagement de Tala-Tala, et dont l'âge des chasseurs varie entre 20 à 65 ans. Le plus grand nombre de chasseurs se trouve dans l'intervalle d'âges variant entre 30 à 45 ans et utilisant beaucoup plus les fusils. Ce sont des jeunes mariés sans emplois et cherchant des moyens de survie pour leur famille. La plupart d'entre eux se sont limités au niveau d'instruction primaire, et pratique l'agriculture comme activité principale. Les résultats de cet auteur sont similaires à ceux de la présente étude, à la seule différence au niveau d'instruction où la fréquence relative est élevée pour les chasseurs, enquêtés dans le DCRT, ayant un niveau secondaire.

Les résultats de Dufour, et al. (2013) démontrent que les chasseurs sont avant tout agriculteurs. Il fait remarquer 
aussi que cette activité n'est pas organisée de même que dans la sous-région Ouest-Africaine. Il en est de même pour les chasseurs de la région forestière de Kisangani.

Par contre Mpamu (2010) a constaté les mêmes tendances que nous. En effet, il a démontré que la chasse est une activité purement masculine. Cet auteur explique que la filière de viande de brousse est animée à Kinshasa ainsi que dans d'autres pays du bassin du Congo par des femmes qui pratiquent le commerce en détail (74,1\%), les hommes quant à eux sont plus concentrés sur la chasse et la pratique de la vente en gros du gibier $(25,8 \%)$. Il ajoute que l'usage du fusil demeure l'une de principales méthodes de chasse $(29,3 \%)$ suivi du piégeage $(24,1 \%)$. Nebesse (2016), a abouti aux résultats selon lesquels les chasseurs de Basukwambula à Baego utilisent souvent le calibre 12 de la fabrication artisanale que les pièges à nylon.

Les résultats de Ayaya (2012) ont mis en évidence le fait que la majorité des chasseurs sont des jeunes mariés ; ils considèrent la chasse comme le moyen le plus direct et rapide d'avoir de l'argent liquide. Pour les pygmées, la chasse est une activité principale alors qu'elle est exercée en seconde position par les bantous. Trois types de techniques de chasse ont été identifiés: les pièges, le filet avec chien, flèches (arbalète). Il signale que la population de Bandisende parait ne pas utiliser le fusil pour la chasse. Les chasseurs Bantous ont un niveau d'étude primaire ou secondaire. Nathalie V. et al. (2010), rapportent que la population dans le milieu rural, en Afrique Centrale, en général et les chasseurs ne sont pas instruits. Ces résultats s'apparentent aux nôtres vu que la majorité d'enquêtés savait lire et écrire mais sans diplômes. La seule différence relevée de l'étude d' Ayaya est le fait que le fusil paraît ne pas être utilisé par les chasseurs de Bandisende.

\section{De l'apport de la chasse dans la survie des chasseurs villageois}

\subsection{De l'apport de la chasse à la consommation des} ménages

L'estimation de la fréquence hebdomadaire de consommation de gibier, pour la plupart de nos enquêtés, a été de 1 à 3 fois la semaine. Les produits de chasse sont considérés comme principale source des protéines animales, d'autant plus que peu de ménages pratiquent l'élevage.

Ces résultats rencontrent ceux de Dufour, et al. (2013), qui estiment que plus de $70 \%$ des ménages enquêtés dans toutes les régions de leur recherche, disent consommer de la viande de brousse une à trois fois par semaine. Alors qu'Endezoumou (2012.) estime une fréquence de consommation moyenne hebdomadaire de 5 jours la semaine.
La consommation de viande par les chasseurs-cueilleurs de la forêt d'Ituri au Nord-Est de la République Démocratique du Congo est estimée à 0,16 kg/personne/jour (Wilkie et Carpenter, 1999). Les populations urbaines au Gabon, en RDC et en RCA consomment en moyenne seulement 0,013 $\mathrm{kg} /$ personne/jour, moins de $10 \%$ de la quantité consommée par les chasseurs-cueilleurs en forêt. Ce qui corrobore nos résultats en démontrant cette caractéristique de la viande de brousse à contribuer à l'alimentation des peuples forestiers.

Par ailleurs Fargeot (2008) démontre que la consommation de viande était plus importante dans les zones urbaines que dans les zones rurales, du fait de la plus forte densité de population. La viande de brousse est largement consommée dans les pays du bassin du Congo, les quantités consommées vont de 30g/personne/jour à $180 \mathrm{gr} / \mathrm{personne} / \mathrm{jour}$ en RDC (Fa et al, 2003). Les résultats de Kambale (2015) démontrent que, à Y angambi, la consommation des animaux sauvages occupe la troisième position après les poissons et les animaux domestiques. Il poursuit en évoquant le fait que la viande de brousse est consommée à Yangambi selon les habitudes alimentaires et selon la disponibilité.

Mpamu (2010) a trouvé aussi que la viande de brousse contribue énormément à la subsistance des populations rurales les plus démunies car accessible.

Les résultats de toutes ces études soutiennent le rôle important de la viande de brousse dans la survie des ménages villageois et l'octroi des revenus telle que démontré par les résultats de cette étude. Ceci confirme l'hypothèse 3 relative à l'apport de la viande de brousse en termes de consommation alimentaire.

\subsection{Du revenu monétaire issu de l'activité de chasse}

Il s'est dégagé dans cette étude, une estimation de revenu mensuel moyen issu de la vente des produits de chasse se fixant à USD 58,18 permettant à un chasseur de répondre aux besoins primaires de son ménage, et celle de la rentabilité de cette activité à 72,19\%. Il sied de souligner que ces valeurs peuvent fluctuer en fonction des périodes de recherche. C'est pourquoi, il est même souhaitable de mener des suivis annuels de chasse afin d'observer le comportement de ces variables pendant des longues périodes. En dépit de cela, la chasse reste une activité qui procure des moyens de subsistance considérables aux populations vivant dans les zones étudiées.

Dans la Réserve de Biosphère de Luki, Toirambe (2002) avait confirmé l'existence d'une véritable entreprise cynégétique dans cette réserve et ses environs avec 16 points de ventes de gibier comptant un effectif de 83 vendeurs (tous des hommes) dont l'âge varie de 20 à 45 ans. Le revenu moyen par vendeur et par semaine était 
évalué à USD 16,14 soit USD 64,56/mois/vendeur. Ces résultats se rapprochent des nôtres.

Les différentes études réalisées à Kinshasa ont montré que les bénéfices tirés par chaque intervenant dans une filière dépendent généralement de la nature du produit et de la loi de l'offre et de la demande. Les résultats de Kabongo (2005) établissent que 93\% des exploitants des PFNLs estiment que leur activité est très rentable car il leur permet tous de satisfaire leurs besoins primaires.

Au Cameroun, les résultats de Lescuyer (2010) ont donné une estimation d'un revenu annuel entre 25000 et 35000 FCFA par chasseur dans plusieurs sites de la région du Sud, et autour de 31000 FCFA dans la commune de Mbang à l'Est. Toujours dans la région de l'Est mais autour de la Réserve du Dja, Ekodeck (2003) estimait le revenu annuel des chasseurs à environ 80000 FCFA par personne. Alors que dans deux sites de la région Sudouest du Cameroun, Willcox \& Nambu (2007) parviennent à une estimation comprise entre 52000 et 85 000 FCFA. Au Gabon (Coad et al., 2010) et en Guinée Equatoriale (Kümpel et al., 2010) ont observé que la majeure partie des revenus issus de la chasse est utilisée par les hommes pour des achats d'alcool et de cigarettes, permettant notamment de renforcer le lien social avec les parents et amis, au Dja (Solly, 2004) comme dans le Sudouest (Wright \& Priston, 2010, cités par Lescuyer, 2010). Quand bien même, cette activité ne permet pas une accumulation de richesse, mais elle permet aux chasseurs vivant dans le DCRT et ailleurs de répondre aux multiples besoins essentiels de base pour leurs familles: scolarisation des enfants, payement des frais d'hospitalisation en cas de maladie, etc.

Dans la zone d'emprise de l'UFA, les résultats d'Endezoumou (2012) démontrent que le commerce de la viande de brousse vient après l'activité forestière industrielle. En effet pour les chasseurs de cette zone, l'activité de chasse leur procure des revenus contribuant à leur bien-être. Ainsi cet auteur a estimé le revenu mensuel moyen à 24 161,94 FCFA dans la zone de Tala-Tala. Cependant, ce revenu est estimé entre 19 082,17 et 28 530,61 FCFA dans le district de Mokéko, 12 783,5 et 17 284,55 FCFA à Ngbala et 28720 et 32586 FCFA dans le district de Sembé. Ces résultats sont un peu différents de ceux obtenus par Noudjieu (2005) lors d'une étude similaire dans l'UTO Campo-Ma'an qui ont établi un revenu mensuel variant entre 3092 et 12735 FCFA. Les grandes valeurs observées sur les écarts types, démontrent la grande variabilité qui existe entre les différents revenus des répondants.

Dufour, et al. (op.cit.) démontrent que la chasse revêt un caractère commercial important. C'est une source d'échanges financiers tant pour les populations rurales que pour certaines populations urbaines. Mpamu (2010) confirme cette observation en élucidant le fait que la filière permet une circulation monétaire importante, dans le sens villes vers campagne, qui équilibre les besoins en numéraire des ruraux, pour financer la santé, l'éducation, les impôts ou l'achat des équipements de base (matériel agricole, matériel de construction) (Feer, 1996).

En RDC, les communautés affectent une part importante de la viande de brousse (90\%) à la vente en vue de trouver des moyens financiers suffisants et le reste $(10 \%)$ est réservé pour la consommation (De Merode et al., 2004). Ce fait a été observé aussi par Nebesse (2016). En effet la poursuite par les chasseurs d'un but lucratif a été observée sur l'axe routier de Kisangani-Ituri. C'est d'ailleurs l'explication du recours prononcé à la technique d'arme à feu jugée plus rentable par ces derniers. C'est comme ça que Kümpel et al. (2010) disent que les chasseurs trouvent flou de faire une différence entre chasse de subsistance et chasse commerciale, étant donné que la viande de brousse contribue à la fois à la consommation et aux revenus.

Fa \& Brown (2009) démontrent que l'activité de chasse joue un rôle essentiel durant les périodes difficiles d'un point de vue alimentaire ou financier. Sur le plan financier, elle permet de tirer des moyens financiers susceptibles de couvrir des dépenses urgentes comme la scolarité ou les soins de santé.

De par ces résultats, il ne cesse d'être mis en exergue l'importance économique de l'activité de chasse pour les riverains des forêts. En effet cette dernière est exprimée par des revenus monétaires non négligeables permettant aux chasseurs de faire face aux besoins quotidiens de leurs foyers. Ces résultats ne font que corroborer ceux démontrés par cette recherche et de ce fait, confirment l'hypothèse 3 relative à l'apport de la chasse dans la survie des chasseurs vivant dans le DCRT.

\section{CONCLUSION}

La viande de brousse reste une source importante des moyens de subsistance pour les populations forestières en région de Kisangani. Elle occupe une place de choix vu son apport sur le plan socioéconomique dans le quotidien des ménages de ces populations. Cependant l'exploitation de cette ressource doit se faire conformément aux textes et mesures de gestion mises en place par l' autorité. En effet, cette étude socioéconomique de la chasse villageoise a été initiée dans le souci d'appréhender toutes ces considérations.

Décrire le profil des acteurs et révéler les aspects socioéconomiques de la chasse villageoise dans l'ancienne Province Orientale tel a été l'objectif principal de cette recherche.

Dans la poursuite de cet objectif, des enquêtes par entretiens semi-structurés auprès des chasseurs répartis dans différentes localités sur les routes Ituri et Buta, des observations directes sur le terrain ont été réalisées. La 
technique documentaire a été utile pour la littérature relative au thème de recherche. Le traitement des données était facilité par Microsoft Excel 2010 et l'analyse des coûts-bénéfices. Cette recherche a abouti aux résultats ciaprès :

- Pour le profil des chasseurs, ils sont tous hommes, répartis dans l'intervalle d'âge allant de 20 à 69 ans. Ils sont issus de différentes ethnies de la contrée mais aussi issues des localités voisines. Comme statut matrimonial, la majorité d'entre eux est marié et responsable de ménage. Concernant le niveau d'instruction, on compte parmi eux des analphabètes, des primairiens et quelques diplômés.

- Il ressort que l'arme à calibre 12 est le moyen le plus utilisé avec $80.67 \%$. Elle est souvent associée aux pièges, aux chiens pour améliorer le rendement.

- La consommation de la viande de brousse intervient 1 à 3 fois/semaine dans la plupart des ménages des chasseurs enquêtés, et l'estimation de revenu mensuel moyen tiré par un chasseur se fixe à USD 58,18 lui permettant ainsi de répondre aux multiples besoins de base; la rentabilité de la chasse était estimée à $72,19 \%$.

Ces résultats confirment toutes les hypothèses qui ont été émises pour cette recherche.

\section{REMERCIEMENTS}

Un grand merci est adressé au CIFOR pour avoir financé les recherches sur terrain ainsi que les programmes de formation de deux Masters co auteurs de ce papier à travers le projet FCCC. Nous saluons les efforts qu'il ne cesse de fournir pour former la jeunesse congolaise élite de demain.

\section{REFERENCES}

[1] Ayaya, I., 2012. Pratique de chasse et caractéristiques des prélèvements de viande de brousse pendant la saison des pluies. Cas de village de Bandisende dans la Réserve de faune à Okapi en Ituri (R.D. Congo) (Mémoire de Master, inédit). Université de Kisangani, Kisangani.

[2] Bahuchet, S., 2000. De la forêt au marché : le commerce de gibier au sud Cameroun. L'Homme et la forêt tropicale. Marseille : Société d'écologie humaine $15 \mathrm{p}$.

[3] Basa D. , Casimir Nebesse , Consolate Kaswera K, Judith Tsongo , Sylvestre Gambalemoke , Benjamin Dudu A, Lelo-Di-Makung, 2017. Bush meat sold on the markets in Kisangani: analysis addressed to the right on species conservation in the Democratic Republic of the Congo . International Journal of Environment, Agriculture and Biotechnology (IJEAB) Vol-2, Issue-2, Mar-Apr- 2017 http://dx.doi.org/10.22161/ijeab/2.2.9 ISSN: 24561878 www.ijaems.com Page 624

[4] Belembo, M., Danadu, M., Gambalemoke, M., Gembu, T., Kaswera, K., Wetshi, L., Katuala, G.B., et Dudu, A. M., 2003. Evolution de l'exploitation du gibier mammalien à Kisangani (R. D. C) De 1976 à 1997. Ann. Fac. Sci. Unikis. vol.12, pp. 303 - 314.

[5] Consolate Kaswera Kyamakya, Nebesse Mololo, Bashonga Bishobibiri Alexis, 2017. RDC :Faune sauvage menacée par le commerce de la viande de brousse. Cebios Policy brief, nr 9, Bruxelles.

[6] Consolate Kaswera Kyamakya , Dudu Akaibe , Jean-Léon Kambale , Erik Verheyen ,et Koto-teNyiwa Ngbolua , 2016: Pression de chasse sur Petrodromus tetradactylus tordayi (Thomas, 1910 : Mammalia) dans six villages des environs de la Réserve Forestière de Yoko (Province de la Tshopo, RD Congo) . International Journal of Innovation and Scientific Research ISSN 2351-8014, Vol. 26 No. 1 A, pp. 182-189.

[7] De Merode A , Katherine Homewood B , Guy Cowlishaw A.,2004. The value of bushmeat and other wild foods to rural households living in extreme poverty in Democratic Republic of Congo. Biological Conservation 118 (2004) 573-581.

[8] Dufour, S., Bikouyah, G., Gautier, M., Nganga, P.Y., Ohlsen, A., Bah, C., Ouendeno, B.D.F., Ansoumane, Moussa, Gamys, A., Aïssatou. (2013). Etude de la chasse et de la filière gibier dans le corridor du Chemin de fer-Projet SIMANDOU/RioTinto, 217p.

[9] Endezoumou (2012). La chasse villageoise dans l'unité forestière d'aménagement Tala-Tala au Nord de la RDC. (Mémoire d'Ingénieur, inédit), Université de Dschang, Cameroun, 92p.

[10]Fa, J.E., Currie, D. \& Meeuwing, J. (2003). Bushmeat and food security in the Congo basin: linkages between wildlife and people's future. Environment conservation, 30 (1): 71-78.

[11]Fargeot, C. (2008). Le commerce de la viande de chasse en Afrique Centrale. Etude d'un marché-porte : le PK 12 à Bangui (RCA).

[12] ICCN/PO (2012). Délimitation du domaine de chasse de Rubi-Tele, Rapport technique Inédit.

[13]Feer, F. (1996). Les potentialités de l'exploitation durable et de l'élevage du gibier en zone forestière tropicale. L'alimentation en forêt tropicale. Interactions bio culturelles et perspectives de développement. H. C. M. UNESCO, Paris, 2, 10391060.

[14] Kabongo, S. (2005). Modalités d'exploitation des ressources naturelles en milieu périurbain: cas de la Commune de N'djili, Facagro, Université de Kinshasa, Kinshasa. 
[15] Kambale (2015). La Consommation de la Viande de Brousse à Yangambi (Tshopo, RDC) (Mémoire de Master inédit), Université de Kisangani, Kisangani.

[16]Laurance, W.F., Croes, B. M., Tchignoumba, L., Lahm, S.A., Alonso, A., LEE, M.E., Campbell, P., Ondzeano, C. (2006). -Impacts of roads and hunting on Central African rainforest mammals. Conservation Biology, 20, 1251-1261.

[17]Lescuyer, G. (2010). Importance économique des produits forestiers non ligneux dans quelques villages du Sud-Cameroun. Bois et Forêts des tropiques, 16.

[18]Loi $\mathrm{n}^{\circ}$ 82-002 du 28 Mai 1982 portant Réglementation de la chasse en RDC.

[19]Mpamu, H. (2010). Etude de la filière de commercialisation de la viande de brousse à Kinshasa (Mémoire de Licence, inédit). Université de Kinshasa, Kinshasa.

[20] Mokuinema, B., Bambu, L., Boundawana, Y. \& Kwazime, N. (2012). Enquêtes socioéconomiques et environnementales auprès des populations riveraines du Parc National de la Maiko. Rapport inédit, Frankfurt Zoological Society, 79p.

[21] Mutambwe, S. (2010). Revue Nationale sur les Produits Forestiers non Ligneux (PFNL) cas de la RDC. CIFOR.

[22] Nebesse, C. (2016). Caractérisation de la viande de brousse prélevée du village Basukwambula (PK 92) au village Baego (PK147) sur l'axe Kisangani-Ituri» (Tshopo, R.D. Congo) (Mémoire de DES, inédit). Faculté des Sciences, Université de Kisangani, Kisangani, 81p.

[23] Toirambe, B. (2002). Valorisation des produits forestiers non ligneux dans la réserve forestière de Luki (Mémoire de DESS, inédit), ERAIFT, Kinshasa, 161 p.

[24] Kümpel, N.F., Milner-Gulland, E.J., Cowlishaw, G. \& Rowcliffe, J.M. (2010). Assessing sustainability at multiple scales in a rotational bushmeat hunting system. Conservation Biology, 24 (3), 861-871.

[25] Pro-Routes (2013). Evaluation préliminaire des appuis à l'ICCN en matière de gestion participative des aires protégées, Kinshasa-RDC.

[26] Nathalie Van Vliet, Robert Nasi, Katharine Abernathy, Christian Fargeot, Noelle F Kumpel, Anne Marie Ndoung Obiong, Stéphane Ringuet, 2010. Le role de la faune dans le cadre de la sécurité alimentaire en Afrique Centrale , une ménace pour la biodiversité. EDF, 123-136.

[27] Wilkie, D.S. \& Carpenter, J.F. (1999). Bushmeat Hunting in the Congo Basin: An Assessment of Impacts and Options of Mitigation. Biodiversity and conservation, 8.1999 Kluwer Academic Publishers. Netherlands. pp. 927-955.
[28] Wilkie, D.S., Starkey, M., Abernethy, K., Nstame Effa, E.N., Telfer, P. \& Godoy, R. (2005). Role of prices and wealth in consumer demand for bushmeat in Gabon, Central Africa. Conservation Biology, 19 (1), 268-274.

[29] Willcox, A.S. \& Nambu D.M. (2007). Wildlife hunting practices and bushmeat dynamics of the Banyangi and Mbo people of Southwestern Cameroon. Biology Conservation, 134, 251-261.

[30] Wright, J.H. \& Priston, N.E.C. (2010). Hunting and trapping in Lebialem Division, Cameroon: bushmeat harvesting practices and human reliance. Endangered species research, 11, 1-12. 